\title{
The effect of complex solvents on the structure and dynamics of protein solutions: The case of Lysozyme in trehalose/water mixtures
}

\author{
Pavan K. GhattyVenkataKrishna ${ }^{1, a}$ and Gustavo A. Carri ${ }^{2, b}$ \\ 1 Computational Biology and Bioinformatics Group, Oak Ridge National Laboratory, Oak Ridge TN 37830, USA \\ 2 Department of Polymer Science and The Institute of Polymer Science and Polymer Engineering, The University of Akron, \\ Akron OH 44325-3909, USA
}

Received 26 July 2012 and Received in final form 15 October 2012

Published online: 14 February 2013

(c) The Author(s) 2013. This article is published with open access at Springerlink.com

\begin{abstract}
We present a Molecular Dynamics simulation study of the effect of trehalose concentration on the structure and dynamics of individual proteins immersed in trehalose/water mixtures. Hen egg-white Lysozyme is used in this study and trehalose concentrations of $0 \%, 10 \%, 20 \%, 30 \%$ and $100 \%$ by weight are explored. Surprisingly, we have found that changes in trehalose concentration do not change the global structural characteristics of the protein as measured by standard quantities like the mean square deviation, radius of gyration, solvent accessible surface area, inertia tensor and asphericity. Only in the limit of pure trehalose these metrics change significantly. Specifically, we found that the protein is compressed by $2 \%$ when immersed in pure trehalose. At the amino acid level there is noticeable rearrangement of the surface residues due to the change in polarity of the surrounding environment with the addition of trehalose. From a dynamic perspective, our computation of the Incoherent Intermediate Scattering Function shows that the protein slows down with increasing trehalose concentration; however, this slowdown is not monotonic. Finally, we also report in-depth results for the hydration layer around the protein including its structure, hydrogen-bonding characteristics and dynamic behavior at different length scales.
\end{abstract}

\section{Introduction}

Understanding anhydrobiosis in Tradigrades and Nematodes is a first class challenge in scientific research. Indeed, it has been a topic of intense interest and research in the scientific community for three decades now [1-3]. In anhydrobiosis, living organisms are frozen in a state where their biological activities are almost completely halted. This dormant state is a function of the environment surrounding the organism. As long as the environment remains hostile to the organism's normal functioning as a being, this dormant state is preserved. As the environment becomes more hospitable, biological activity is restarted, thus taking the organism to an active state. Depending on the species, an increase in the amount of sugars like trehalose and sucrose which have been reported mainly in invertebrates and plants, respectively, or alcohols like glycerol has been identified as the main reason for the onset of anhydrobiosis [1]. This insight has already found valuable practical applications in the formulations of dry vaccines that reactivate once they are injected into the body. Since

a e-mail: pkc@ornl.gov

b e-mail: gac@uakron.edu many current vaccines are protein-based, a plethora of scientific studies about the behavior of proteins in various kinds of complex, including bio-preserving, solvents have followed. The study we present in this paper contributes to this field by investigating the effect of trehalose concentration (in water) on the structure and dynamics of globular proteins.

It has been established that trehalose is one of the most effective substances (biopreservants) for increasing the stability of proteins [1-7]. The presence of four hydroxyl groups on each ring allows for the formation of a large number of hydrogen bonds with the surrounding water [8] and protein surface. Water molecules usually form a spanning hydrogen-bonded network around the protein [9]. However, this network is destructured by the presence of trehalose [8]. In addition, it is also well documented that proteins require a minimum amount of hydration to be biologically active $[10,11]$. The destructuring $[8,12]$ effect of trehalose leads to a decrease in the hydration water rendering the protein inactive. Similarly, the presence of trehalose hinders the formation of ice, thus protecting the protein from liquid-water-to-ice transition induced damage. 
Another important aspect of protein solutions is their dynamic behavior. In this case, understanding the structure and dynamics of the protein-solvent interface is critical for a comprehensive understanding of the dynamic behavior of proteins. Indeed, it is well known that the dynamics of proteins is slaved to the one of the surrounding solvents [13-17]. In the case of pure trehalose, the dynamic coupling between the protein and the solvent has been studied by Dirama et al. [18]. In this study, the authors concluded that the protein atoms at the core of the protein can relax at a faster or slower rate than surface atoms depending on the dynamics of the surrounding medium which, in turn, is controlled by temperature. The dynamic information of the medium is transfered to the protein through the interface thus providing a compelling argument for its study. The results of Dirama et al. [18] show that the dynamics of proteins is heterogeneous and slaved to the solvent. In general, Frauenfelder et al. [15] have argued that the effects of hydration and solvent viscosity are mutually exclusive and important for understanding the dynamics of proteins. Thus, the case of trehalose-water binary mixtures at various concentrations provides useful insights into the effect of solvent on the structure and dynamics of the protein-solvent interface and its consequences on protein dynamics.

Computer simulation studies for a protein-water system in the 100 ps time scale have shown that dehydration and a decrease in temperature have the same effect on protein dynamics [19]. Indeed, a decrease in the temperature of the system reduces solvent translational motions, thus increasing the lifetimes of protein-water hydrogen bonds and slowing down protein dynamics. Similarly, an increasing degree of dehydration makes the internal dynamics of the protein less activated [20]. In this paper we discuss similar findings, i.e. an increasing concentration of trehalose leads to higher dehydration close to the protein surface and, consequently, to longer hydrogen bond lifetimes and slower protein dynamics. This effect, which we study at low trehalose concentration in this paper, is expected to become more significant as the concentration of trehalose increases.

The behavior of proteins in pure water $[19,21-56]$ and pure trehalose $[18,57]$ has been studied extensively using computer simulation methods. In the particular case of globular proteins immersed in trehalose-water binary mixtures Lins et al. [58] and Lebret et al. [56] have reported studies of Lysozyme in various trehalose-water mixtures. Indeed, Lins et al. studied the behavior of Lysozyme in a $18 \%$ by weight trehalose-water binary mixture [58] and concluded that trehalose helps enhance the stability of the protein by forming a network of trehalose molecules around the protein thereby trapping a layer of water molecules between the trehalose network and the protein. Lerbret et al. [56] studied Lysozyme in water-sugar (trehalose/maltose/sucrose) solutions of 37, 50 and $60 \%$ by weight of sugar. Although the aim of the study was to understand the differences in the behaviour of various sugars, interesting insights were obtained for a protein in water-trehalose binary mixtures. Trehalose was found to be preferentially excluded from the protein surface and the relaxation time of the protein increased with increasing amounts of trehalose.

More recently, differential scanning calorimetric studies showed that at low hydration, the denaturation temperature of proteins in protein-trehalose-water ternary mixtures closely followed the glass transition temperature of corresponding trehalose-water binary mixtures $[59,60]$. Trehalose has also been called a "chemical chaperone" [61] responsible for preserving the structure of proteins in dried as well as lightly hydrated systems [62,63].

In this paper we focus on the changes induced in the equilibrium and dynamic properties of the proteintrehalose-water mixtures during the initial build-up of trehalose. This is important for various reasons. First, we are interested in clarifying the equilibrium structure of the solvent next to the protein surface. Among the various questions we want to address, we are interested in understanding the conditions for the formation of the trehalose network surrounding the protein suggested by Lins et al. Second, as stated before, hydrogen bonds play a fundamental role in protein dynamics. However, in the case of mixtures, various kinds of hydrogen bonds can form and their effects on protein dynamics and solvent structure must be quantified and studied. Third, the local viscosity of the solution around the protein increases due to changes in the local structure of water, solvent composition and the enhanced lifetimes of the hydrogen bonding network. It is of interest to study such increases in viscosity due to decreases in water of hydration. It is this increase in viscosity which is supposed to curb the internal motions of proteins therefore contributing to its bio-preservation. For this work we have chosen hen egg-white Lysozyme (HEWL PDB 193L) [64] as the model protein and we will address the first two topics leaving the third one for a future publication.

Desiccation triggers the production of trehalose at an elevated rate in some organisms [65]. Our interest here is in the early stages of desiccation where the concentration of trehalose is relatively low. Based on previous work, it is reasonable to expect the protein to be preferentially hydrated at higher trehalose concentrations [56]. Also, at higher trehalose concentrations, movement of water is damped which will necessitate simulation time scales much longer than those employed here to attain equilibrium. It is due to these reasons that we confine this study to 10,20 and $30 \%$ wt trehalose in the binary mixtures.

This paper is organized as follows. In sect. 2 we describe the details of the computer simulation protocol used in this study. Section 3 contains the results of our study and their discussion. Finally, we present the conclusions in sect. 4 followed by the appropriate acknowledgments.

\section{Simulation protocol}

In this study we employed the AMBER 9.0 Molecular Dynamics package [66] with the ff99SB [67] force field to model the protein. Trehalose was model with the General Amber Force Field (GAFF) [41]. The structure and partial charges were obtained from the work of Dirama 
et al. [18]. We modeled water with the TIP3P rigid water model [51].

We built and equilibrated the trehalose-water mixtures as follows. First, a single trehalose molecule was surrounded with the appropriate number of water molecules to make unit boxes with 10, 20 and 30 percent trehalose by weight. Afterward, these unit boxes were copied multiple times to create a larger box for each trehalose concentration.

This box was first heated from 0 to $200 \mathrm{~K}$ while being subjected to a pressure of $10 \mathrm{~atm}$ over a period of $200 \mathrm{ps}$. This was done to remove any voids in the box. Once the boxes attained near cubic dimensions without voids, the pressure was reduced from $10 \mathrm{~atm}$ to $1 \mathrm{~atm}$ in 3 decrements of $3 \mathrm{~atm}$ each while maintaining the temperature at $200 \mathrm{~K}$ and running each simulation for $100 \mathrm{ps}$ in the NPT ensemble.

This was followed with two isobaric simulations; in each of them the temperature was increased by $50 \mathrm{~K}$ over a period of $100 \mathrm{ps}$ and the pressure was $1 \mathrm{~atm}$. This resulted in boxes with a homogeneous distribution of trehalose in water which were later equilibrated for $500 \mathrm{ps}$ in the NPT ensemble at $1 \mathrm{~atm}$ and $300 \mathrm{~K}$.

In the case of pure trehalose, 600 molecules were confined in a cubic box which was first heated from 0 to $500 \mathrm{~K}$ while being subjected to a pressure of $10 \mathrm{~atm}$ over a period of $200 \mathrm{ps}$. This removed the voids present in the box. The pressure was then reduced from $10 \mathrm{~atm}$ to $1 \mathrm{~atm}$ in 3 decrements of $3 \mathrm{~atm}$ each while maintaining the temperature at $500 \mathrm{~K}$ and running each simulation for $100 \mathrm{ps}$ in the NPT ensemble. This was followed with two isobaric simulations; in each of them the temperature was decreased by $100 \mathrm{~K}$ over a period of $200 \mathrm{ps}$. Afterward, the simulation box was equilibrated for $500 \mathrm{ps}$ in the NPT ensemble at $1 \mathrm{~atm}$ and $300 \mathrm{~K}$.

The protein was placed in the geometric center of the pre-equilibrated boxes of 10, 20, 30 and 100 percent by weight of trehalose in water at $300 \mathrm{~K}$ and $1 \mathrm{~atm}$. All solvent molecules within $2 \AA$ from the protein surface were removed and additional solvent molecules were added/removed to keep the concentration as desired. This produced boxes with 48(8270), 64(4864), 116(5104) and 471(0) trehalose(water) molecules for the 10, 20, 30 and 100 percent by weight trehalose-water mixtures, respectively.

The partial mesh Ewald (PME) [68] procedure was used to calculate long-range electrostatic interactions and the 6-12 Lennard-Jones potential was used to calculate van der Waals interactions. A cutoff of $10 \AA$ was used for non-bonded van der Waals interactions and was also the cutoff used for the calculation of Ewald's standard direct sum; corrections are taken in to account via the reciprocal sum. The equations of motions were integrated using the leap-frog Verlet algorithm with a step size of $1 \mathrm{fs}$. The Berendsen barostat was used to maintain constant pressure [69] and Langevin thermostat with a collision frequency of $1 \mathrm{ps}^{-1}$ was used for temperature control [70].

The equilibration of the final boxes was done following a four step process. In the first step, the energy minimized boxes were heated from 0 to $400 \mathrm{~K}$ under constant volume conditions over a period of $500 \mathrm{ps}$. Second, the system was cooled to $300 \mathrm{~K}$ over a period of $100 \mathrm{ps}$ also under constant volume conditions.

During the first two steps the motions of the atoms in the protein were restrained using a harmonic potential on each atom with a spring constant of $2 \mathrm{Kcal} / \mathrm{mol} \AA^{2}$. Third, the restraints on the protein were removed and the system was simulated for $100 \mathrm{ps}$ under constant volume conditions. Fourth, the equilibration was completed with a $100 \mathrm{ps}$ of NPT simulation. After equilibration, $50 \mathrm{ps}$ of data were collected every $10 \mathrm{fs}$ and then $20 \mathrm{~ns}$ every ps at $300 \mathrm{~K}$ and $1 \mathrm{~atm}$ under NPT conditions. The first $50 \mathrm{ps}$ were collected to study the short-time dynamics of protein and solvent using the Incoherent Intermediate Scattering function.

In the control case, the protein was solvated with 5138 TIP3P rigid water molecules. The system was heated from 0 to $250 \mathrm{~K}$ at constant volume for $100 \mathrm{ps}$ followed by $100 \mathrm{ps}$ of constant pressure and constant temperature simulation at $250 \mathrm{~K}$. This was followed by $1 \mathrm{~ns}$ of heating from 250 to $300 \mathrm{~K}$ at constant pressure. $20 \mathrm{~ns}$ of data were then collected at $300 \mathrm{~K}$ and $1 \mathrm{~atm}$ under NPT conditions.

\section{Results and discussions}

\subsection{Protein structure}

The first step in quantifying the effect of complex solvents on the structure of proteins involves the evaluation of the root mean-squared deviation (RMSD) of the protein atoms with respect to their positions in the crystal configuration. The RMSD provides a measure of the average degree of deformation induced on the protein by the surrounding solvent when the translation of the center of mass of the protein and rigid-body rotations are removed from the trajectories. We evaluated the RMSD [71] for all the protein atoms and for the backbone atoms only, i.e. $\alpha$-carbon, carboxyl carbon and oxygen, and nitrogen during the last $10 \mathrm{~ns}$ of the simulation. Table 1 shows the results. The RMSD for all the systems studied is small and shows no statistically significant correlation with the concentration of trehalose. The RMSD computed using all atoms in the protein displays a maximum at $20 \%$ trehalose concentration. This peak is not observed when only backbone atoms are used to compute the RMSD which indicates that the maximum in the RMSD is a consequence of atoms present on side groups. Thus, no significant change in the structure of the protein occurs at $20 \%$ trehalose concentration. However, at $100 \%$ trehalose concentration the RMSD of the backbone atoms is larger than for the other systems studied. This indicates a larger degree of deformation of the protein. Still, the RMSD is below $2 \AA$, so it is small. In all the cases studied the RMSD is smaller than $2 \AA$ indicating a very small deviation of the protein structure from its crystalline configuration. These findings are in very good quantitative agreement with previous computational studies done at different trehalose concentrations $[56,58]$. 
Table 1. RMSD of the protein atoms with respect to the crystal structure for all and backbone atoms (in $\AA$ ), radius of gyration (in $\AA$ ) and solvent accessible surface area $\left(\right.$ in $\AA^{2}$ ). Numbers in parentheses are the errors as measured by the standard deviation.

\begin{tabular}{lllll}
\hline \hline$\%$ trehalose & RMSD (all atoms) & RMSD (backbone) & $\sqrt{R_{g}^{2}}$ & SASA \\
\hline 0 & $1.579(0.095)$ & $0.945(0.118)$ & $14.269(0.077)$ & $7293(106)$ \\
10 & $1.511(0.103)$ & $0.888(0.085)$ & $14.296(0.070)$ & $7331(94)$ \\
20 & $1.766(0.188)$ & $0.894(0.084)$ & $14.287(0.065)$ & $7320(88)$ \\
30 & $1.458(0.125)$ & $0.907(0.109)$ & $14.317(0.064)$ & $7324(101)$ \\
100 & $1.502(0.038)$ & $1.195(0.038)$ & $13.996(0.043)$ & $6730(45)$ \\
\hline \hline
\end{tabular}

We also calculated the radius of gyration of the protein. Table 1 shows this analysis for all the systems studied. As in the case of the RMSD no statistically significant dependence of $\left\langle R_{g}^{2}\right\rangle$ on trehalose concentration is observed. However, in the case of pure trehalose, $\left\langle R_{g}^{2}\right\rangle$ is smaller than for the other systems. This indicates that the increase in the RMSD of the backbone atoms for the case of pure trehalose is due to the compression of the protein. This is an interesting observation with possible connotations for biopreservation.

Table 1 also shows the solvent accessible surface area of the protein (SASA) which was computed using the Visual Molecular Dynamics package, VMD [26]. The size of the scanning probe was $1.4 \AA$ which is comparable to the size of a water molecule. As in the cases of the RMSD and $\left\langle R_{g}^{2}\right\rangle$, our results show the absence of any statistically significant correlation between SASA and trehalose concentration. However, the SASA decreases for pure trehalose in agreement with the aforementioned compression of Lysozyme under this condition.

To further characterize the effect of trehalose concentration on the structure of Lysozyme, we computed the inertia tensor, I [72], and asphericity, $\Omega$ [73]. Each element of the inertia tensor was computed as follows:

$$
I_{i, j}=\left\langle\frac{\sum_{k=1}^{N} m_{k}\left(\left(r_{k}-g\right)^{2} \delta_{i, j}-\left(r_{k}-g\right)_{i}\left(r_{k}-g\right)_{j}\right)}{\sum_{k=1}^{N} m_{k}}\right\rangle
$$

and the asphericity was computed using the formula

$$
\Omega=\frac{\left(I_{1}-I_{2}\right)^{2}+\left(I_{2}-I_{3}\right)^{2}+\left(I_{3}-I_{1}\right)^{2}}{2\left(I_{1}+I_{2}+I_{3}\right)^{2}},
$$

where $I_{1}, I_{2}$ and $I_{3}$ are the principal moments of inertia of the tensor I. Table 2 shows the principal moments of inertia and $\Omega$. The results clearly show that the principal moments of inertia and asphericity are insensitive trehalose concentration. Moreover, in the case of pure trehalose, we observe a uniform decrease in the principal moments of inertia from their values in the trehalose-water mixtures of about $4 \%$. This decrease does not change the value of the asphericity indicating a uniform compression of the protein. Various energy terms related to the structure of the protein are shown in table 3 . None of the energy terms display a statistically significant correlation with trehalose concentration. However, it is interesting to observe that for the case of pure trehalose the bond energy does not
Table 2. Principal moments of inertia $\left(\AA^{2}\right)$ and asphericity. The errors in these quantities are of the order of 1 to $2 \%$.

\begin{tabular}{ccccc}
\hline \hline \% trehalose & $I_{1}$ & $I_{2}$ & $I_{3}$ & $\Omega$ \\
\hline 0 & 153.51 & 162.95 & 87.68 & 0.031 \\
10 & 154.01 & 163.48 & 88.01 & 0.031 \\
20 & 154.33 & 162.70 & 88.45 & 0.030 \\
30 & 155.31 & 164.03 & 87.34 & 0.032 \\
100 & 148.18 & 157.31 & 84.13 & 0.031 \\
\hline \hline
\end{tabular}

Table 3. Bond, angle and dihedral energies in Kcal $/ \mathrm{mol}$. Numbers in parentheses are the errors as measured by the standard deviation.

\begin{tabular}{cccc}
\hline \hline$\%$ trehalose & Bond & Bond angle & Dihedral angle \\
\hline 0 & $380(16)$ & $929(23)$ & $1353(14)$ \\
10 & $380(16)$ & $932(23)$ & $1355(14)$ \\
20 & $379(16)$ & $929(23)$ & $1354(14)$ \\
30 & $379(16)$ & $929(23)$ & $1354(14)$ \\
100 & $381(16)$ & $967(23)$ & $1411(15)$ \\
\hline \hline
\end{tabular}

change with respect to its values for the other systems but, the energies related to the bond and dihedral angles increase significantly. This indicates that the compression of the protein in pure trehalose is achieved by twisting the dihedral angles and bending the bond angles. Surely, this must generate a significant amount of internal stress in the protein.

The data from tables 1,2 and 3 tell us that when the protein is immersed in pure trehalose it occupies a smaller volume than when it is immersed in pure water or trehalose-water mixtures. The compression generates the bending and twisting of bond and dihedral angles in the protein, respectively. Additionally, the asphericity $\Omega$ shows that the compression is isotropic.

The global structural properties of the protein change little between 0, 10, 20 and 30\% wt trehalose. Possible reason for this is that only above $37 \%$ wt trehalose there exists a precolating hydrogen bond network [56]. Below this limit, although we do not observe large changes globally, there are many interesting local changes as discussed below.

A deeper study of the changes induced in the protein's structure can be carried out by identifying the contributions of the individual domains that comprise the protein. 
Table 4. List of domains in Lysozyme (193L).

\begin{tabular}{ccc}
\hline Domain number & Domain type & Residue group \\
\hline 1 & Loop & $1-4$ \\
2 & Helix & $5-15$ \\
3 & Loop & $16-19$ \\
4 & Helix & $20-22$ \\
5 & Loop & $23-24$ \\
6 & Helix & $25-35$ \\
7 & Loop & $36-42$ \\
8 & Beta & $43-45$ \\
9 & Loop & $46-50$ \\
10 & Beta & $51-53$ \\
11 & Loop & $54-57$ \\
12 & Beta & $58-59$ \\
13 & Loop & $60-79$ \\
14 & Helix & $80-83$ \\
15 & Loop & $84-87$ \\
16 & Helix & $88-100$ \\
17 & Loop & $101-108$ \\
18 & Helix & $109-115$ \\
19 & Loop & $116-119$ \\
20 & Helix & $120-124$ \\
21 & Loop & $125-129$ \\
\hline & &
\end{tabular}

A protein's secondary structure can be divided into $\alpha$ helices, $\beta$-sheets and loops. Using the STRIDE [74] program implemented in VMD [26], residues of the crystal structure of hen egg-white Lysozyme (PDB 193L) [64] were grouped into domains of $\alpha$-helices, $\beta$-sheets and loops. The assignments of the domains are showed in table 4 . Figure 1 reveals that the domains primarily responsible for the shrinkage of the protein are $1,2,8,13,17$ and 18 of which the odd-numbered domains correspond to loops, while domains 2 and 18 correspond to $\alpha$-helices and 8 is a $\beta$-sheet. A visual inspection of the PDB file using VMD showed that all these domains were exposed to the solvent which explains why their SASA are more sensitive to a compression of the protein than the other, more buried domains.

Figure 2 provides a deeper insight at the residue level. The changes in the SASA of individual residues in trehalose-water mixtures is compared with the ones in pure water and shown in figs. 2(a),(b),(c). We note that residue CYS6 is more exposed while CYS127 is more buried. In the crystal structure, the sulfur in residue CYS6, which is part of the CYS6-CYS127 disulfide bond, is on the surface of the protein. As trehalose is introduced into the solvent, this sulfur is more exposed to the solvent consequently pulling CYS127 away from the surface. This contributes to the reduced SASA of CYS127. ARG112 is more buried. This contributes to the decreased SASA of domain 18 observed in all binary solutions studied. ARG61 is more buried in all the solutions. Both ARG61 and ARG112 are on the surface giving them access to the solvent. This result combined with the fact that Arginines

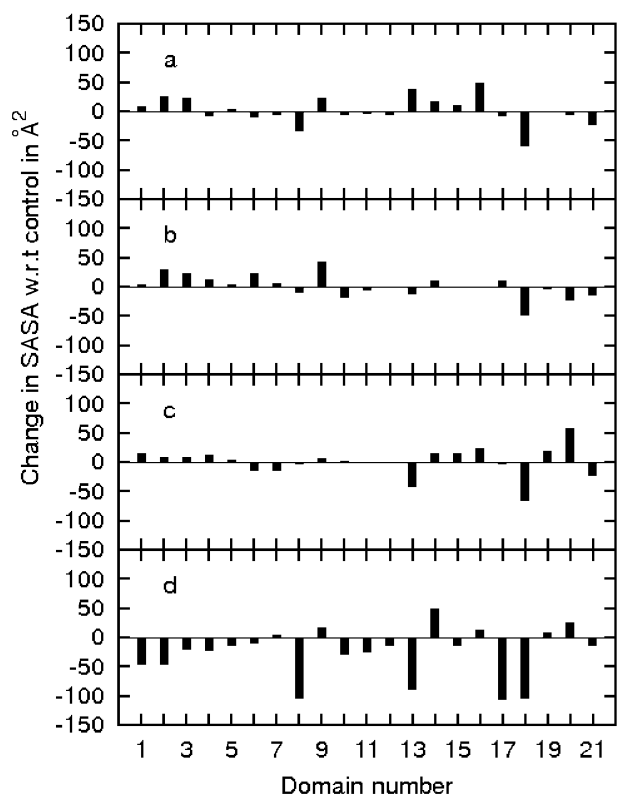

Fig. 1. SASA of each domain (as defined in table 4) of the protein in trehalose-water solutions compared to the protein in pure water. (a) $10 \%$ trehalose- $0 \%$ trehalose. (b) $20 \%$ trehalose- $0 \%$ trehalose. (c) $30 \%$ trehalose- $0 \%$ trehalose. (d) $100 \%$ trehalose- $0 \%$ trehalose.

are polar explains why they are more buried in the presence of a solvent (trehalose-water binary mixture) less polar than water.

When the structure of the protein in pure trehalose is compared to the one in pure water, seven of the eleven Arginines in the protein are more buried. Arginine being a strongly basic residue is typically found on the surface of a protein when immersed in water. However, when it is immersed in trehalose [75], a solvent less polar than water, Arginine tends to reduce the area of contact. The behavior of neutral residues however depends strongly on their spatial neighbors. VAL109, for example, is surrounded by hydrophobic residues which tend to get exposed in pure trehalose causing it to be buried. On the other hand, VAL120 is surrounded by polar residues which tend to be buried in pure trehalose; this leads to the increase in its SASA. The reduction in the SASA of residues ASN44, ARG45 and ASN46 contributes to the decreased SASA of domain 8. A similar effect is seen in domain 13 due to ARG61, domain 17 due to ASN103 and domain 18 due to residues VAL109, TRP111 and ARG112.

\subsection{Protein dynamics}

\subsubsection{Incoherent Intermediate Scattering function}

The overall dynamic behavior of the protein was characterized using the Incoherent Intermediate Scattering function, $I(\mathbf{q}, t)$, which was computed from its definition

$$
I(\mathbf{q}, t)=\frac{1}{N}\left\langle\sum_{j=1}^{N} e^{\left[i \mathbf{q} \cdot\left\{\mathbf{r}_{j}(t)-\mathbf{r}_{j}(0)\right\}\right]}\right\rangle,
$$




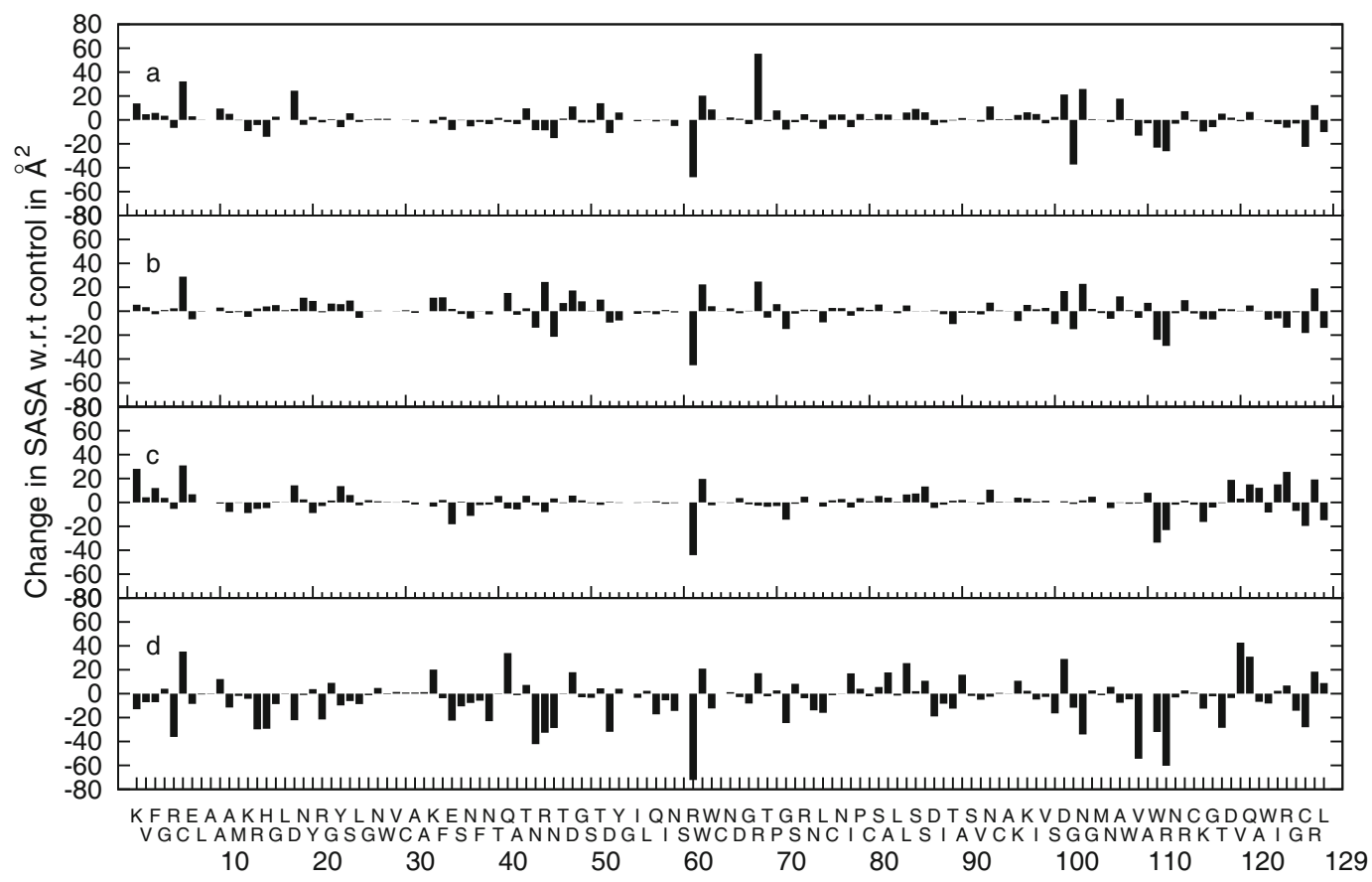

Fig. 2. SASA of each residue in trehalose-water solutions compared to pure water. (a) $10 \%$ trehalose- $0 \%$ trehalose. (b) $20 \%$ trehalose- $0 \%$ trehalose. (c) $30 \%$ trehalose- $0 \%$ trehalose. (d) $100 \%$ trehalose- $0 \%$ trehalose.

where $\mathbf{q}$ is the wave vector (in units of $\AA^{-1}$ ), $t$ is time (in units of ps), $N$ is the total number of atoms used in the evaluation, $\mathbf{r}_{j}(t)$ is the position of the $j$ th atom at time $t$ and the brackets indicate averaging over time origins. For the evaluation of $I(\mathbf{q}, t)$ we used $|\mathbf{q}|=2 \AA^{-1}$, as done in previous studies on this subject $[19,76]$. Furthermore, the results were averaged over 66 different directions of the wave vector, q. The results are shown in fig. 3 . The data for the time interval $0.01-1$ ps were obtained by running a $100 \mathrm{ps}$ long simulation for all the compositions and storing the data after every $10 \mathrm{fs}$. For the rest of the time interval 1 ps -10 ns we employed the protocol described in sect. 2 .

The evaluation of $I(\mathbf{q}, t)$ was done using the hydrogen atoms because of their approximately uniform distribution through out the protein. However, the work by Sokolov and co-workers $[77,78]$ have shown that methyl group rotation is activated at temperatures as low as $100 \mathrm{~K}$ degrees. Thus, we did not consider methyl hydrogens because their contributions would have obscured the true structural relaxation of the protein.

As expected from previous studies [18], we observe in fig. 3 that the $I(\mathbf{q}, t)$ of the protein in pure trehalose decays at a much slower rate than in solutions where water is present; this result is in very good quantitative agreement with previous studies [18]. Moreover, the effect of caging on protein dynamics is clearly depicted and lasts for $5 \mathrm{~ns}$, approximately. At this time trehalose is known to have a secondary structural relaxation [18] which is known to accelerate the dynamics of proteins. Thus, $I(\mathbf{q}, t)$ decreases more rapidly. Note that for proteins in pure trehalose two relaxation processes are clearly identifiable. This is not the case of Lysozyme in trehalose-water mixtures or in pure water. Indeed, the behavior in hydrated solutions follows the one reported before $[19,49]$. For the cases of pure water and trehalose-water mixtures one relaxation process can be identified in the 0.1 ps time scale clearly. However, the second process has been pushed to a timescale outside our time window or has disappeared altogether due to the non-glassy behavior of water and trehalose-water mixtures at room temperature. For the case of pure water, the plot seems to suggest that a second plateau might occur in the tens of $n s$ time scale. Longer simulations are needed to confirm this speculation.

In fig. 3 we see that as the concentration of trehalose increases, the curves move upward showing how the effect of caging is built up. This increase in the concentration of trehalose decreases solvent diffusion around the protein thus creating the cage and slowing down the dynamics of the protein [58]. Curiously, above $2 \mathrm{ps}$, protein relaxes faster in $30 \%$ trehalose than in $20 \%$ trehalose, although the difference is very small. It is reasonable to expect relaxation times to increase with increasing trehalose concentrations. The observed discrepancy might be due to insufficient length of simulations and insufficient time interval between storing trajectories (we stored trajectories every picosecond).

Finally, it is interesting to observe a very uncommon behavior for the Incoherent Intermediate Scattering function. The $I(\mathbf{q}, t)$ for the cases of the trehalose-water mixtures do not show the typical plateau followed by a decay behavior which is usually used to detect relaxation processes. Indeed, these curves show a clear linear dependence on the logarithm of time, i.e. logarithmic decay, over two or three orders of magnitude in time. This is a very uncommon situation since it shows the absence of a characteristic time scale in the decay. 


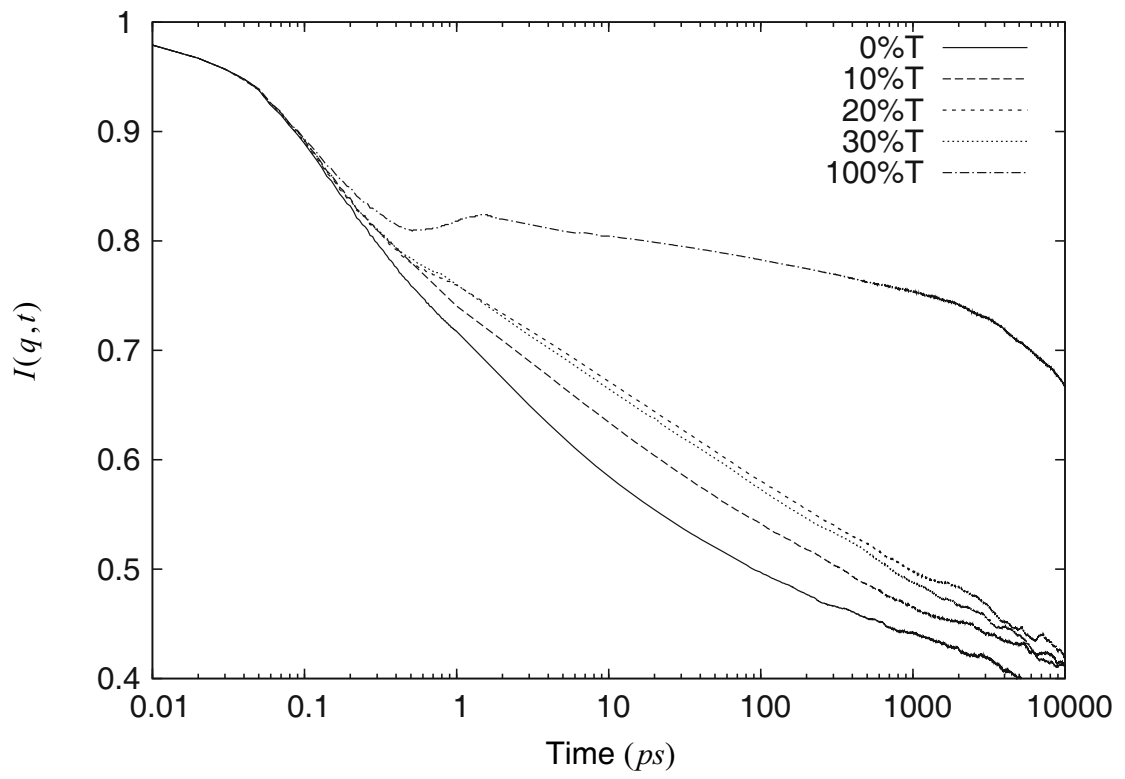

Fig. 3. Intermediate scattering function of non-methyl hydrogen atoms in the protein. At short time scales, protein atoms relax faster in $20 \%$ trehalose than in $30 \%$ trehalose solutions but the trend reverses at longer time scale.

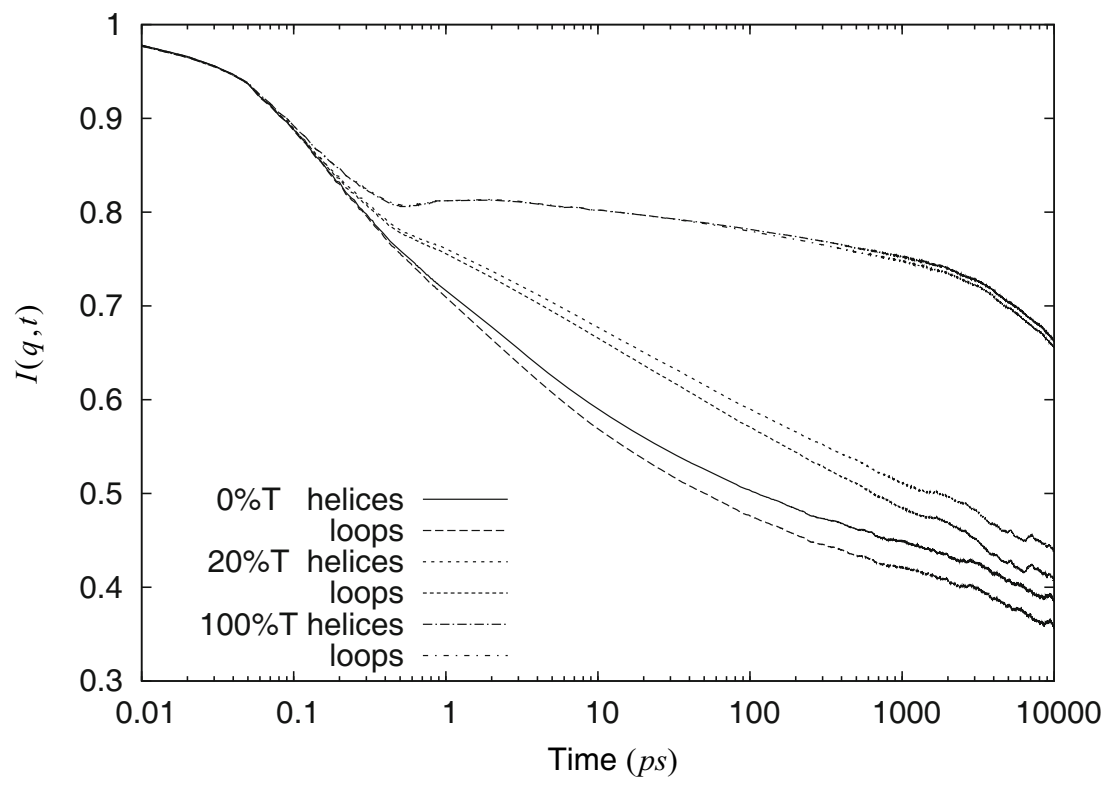

Fig. 4. Intermediate scattering function of non-methyl hydrogen atoms in the protein separated in to loops and helices. Plots for 0,20 and $100 \%$ trehalose have been included for the sake of clarity.

For the purpose of gaining a deeper insight into the dynamic behavior of the protein, we computed $I(\mathbf{q}, t)$ for two different types of domains: loops and helices. The results are shown in fig. 4. For each unary and binary solvent the structural relaxation of loops is faster than the one of helices as it is to be expected because of the intrinsic rigidity of the helical structure. In addition, the logarithmic decay is also observed for each type of domain in the cases of trehalose-water mixtures.

As the process of desiccation progresses and more trehalose is found in the vicinity of proteins, the dynamics of the protein will be more damped and start approaching the limit of pure trehalose. This damping of protein motions as a function of trehalose content can be expected to be non-linear as is the case with many binary mixtures [56]. At a critical trehalose concentration, the protein dynamics can be expected to heavily damped beyond which damping happens only in smaller and smaller steps as the limit of pure trehalose approaches.

\subsubsection{Dynamic cross-correlation map}

A different perspective on protein dynamics can be obtained using the dynamic cross-correlation matrix, 

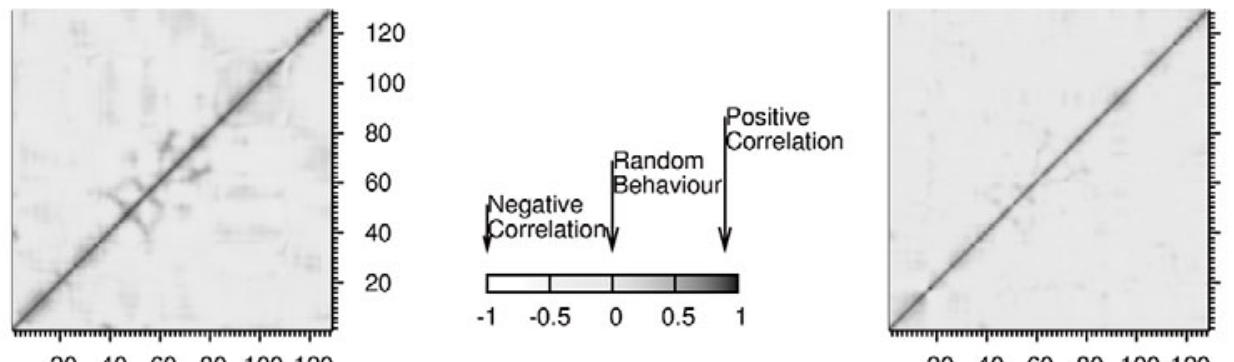

$\begin{array}{llllll}20 & 40 & 60 & 80 & 100 & 120\end{array}$ $0 \%$ Trehalose
$10 \%$ Trehalose

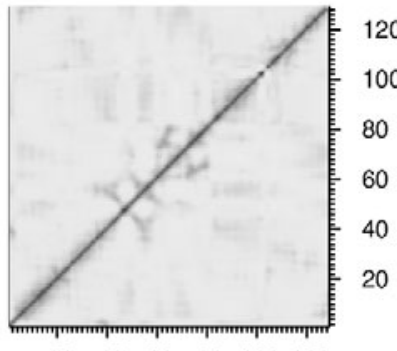

$20 \%$ Trehalose

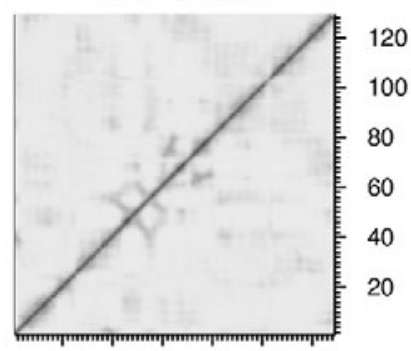

$\begin{array}{llllll}20 & 40 & 60 & 80 & 100 & 120\end{array}$

$\begin{array}{llllll}20 & 40 & 60 & 80 & 100 & 120\end{array}$ $100 \%$ Trehalose $30 \%$ Trehalose

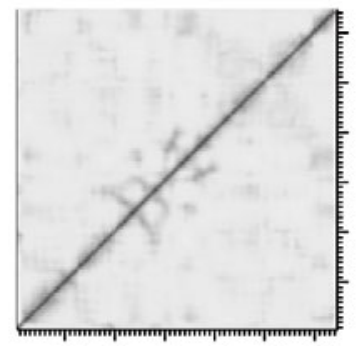

$\begin{array}{llllll}20 & 40 & 60 & 80 & 100 & 120\end{array}$

Fig. 5. DCCM of the $\alpha$-carbons in the protein at different trehalose concentrations.

DCCM. The elements of this matrix quantify the correlation between the motions of different protein atoms and provide information about the effect of solvent on the collective motions in the protein. Such correlations are calculated using the equation [79-81]

$$
\operatorname{DCCM}(i, j)=\frac{\left\langle\Delta r_{i} \cdot \Delta r_{j}\right\rangle}{\left(\left\langle\Delta r_{i}^{2}\right\rangle\left\langle\Delta r_{j}^{2}\right\rangle\right)^{1 / 2}}
$$

where $\Delta r_{i}$ is the displacement of the $i$-th atom from its mean position during the simulation, the dot indicates scalar product, the brackets indicate averaging over the entire simulation, and the possible values of the matrix elements range from 1 to -1 . Calculations based on this method have been successfully employed to identify functional domains [82] and important spatial interactions in proteins [83].

We evaluated the DCCM for the every pair of alpha carbons in the protein and used a gray scale to present the data in a $2 \mathrm{D}$ format shown in fig. 5 . The abscissa and ordinate correspond to residue numbers. Dark gray or black indicates positive correlation and white indicates negative correlation. The diagonal elements equal one since they measure self-correlation. Figure 5 shows a clear distinction between the hydrated and dehydrated cases. The similarities of the DCCM for solutions containing water indicate that the solvent surrounding the protein is essentially the same for all the compositions. Thus, we can safely conclude that it is water that primarily coats the surface of the protein in agreement with the exclusion principle suggested by Gekko and Timasheff $[44,45]$ and the results of Lins et al. [58] and Lerbret et al. [56].

\subsection{Dynamics of the hydration layer}

We define the hydration layer as the layer of solvent that coats the protein and has a thickness equal to $3 \AA$. Many properties of this layer are important and we start by looking at the residence times for water molecules. The basic idea is that if $N$ molecules are in the layer at time 0 , only a fraction of those $N$ molecules will still remain in the layer at time $t$. At longer times, the number of water molecules that were in the layer at $t=0$ reaches a constant value which is the number of bound water molecules. The number of these bound water molecules should reveal interesting information about the effect of trehalose concentration on water molecules close to the protein's surface. For this computation we only used the last $10 \mathrm{~ns}$ of the simulations. The data were first plotted for fourteen different starting points spread evenly between $10 \mathrm{~ns}$ and $16.5 \mathrm{~ns}$, and these plots were later averaged. Figure 6 shows the result for the pure water case.

As can been seen from fig. 6, there are two kinds of water molecules: mobile and bound. To account for the time scale characteristic of the diffusion of the mobile water molecules, we fitted the data with functions of the form

$$
N(t)=n_{\text {bound }}+n_{\text {mobile }} \exp \left(-t / \tau_{\text {mobile }}\right),
$$

where, $n_{\text {bound }}$ and $n_{\text {mobile }}$ are the number of bound and mobile water molecules while $\tau_{\text {mobile }}$ is the diffusion time of the mobile water molecules. At long enough times, even the bound water molecules will be replaced by other water molecules. Thus, the data could be better modeled using the mathematical expression

$$
N(t)=n_{\text {bound }} \exp \left(-t / \tau_{\text {bound }}\right)+n_{\text {mobile }} \exp \left(-t / \tau_{\text {mobile }}\right)
$$




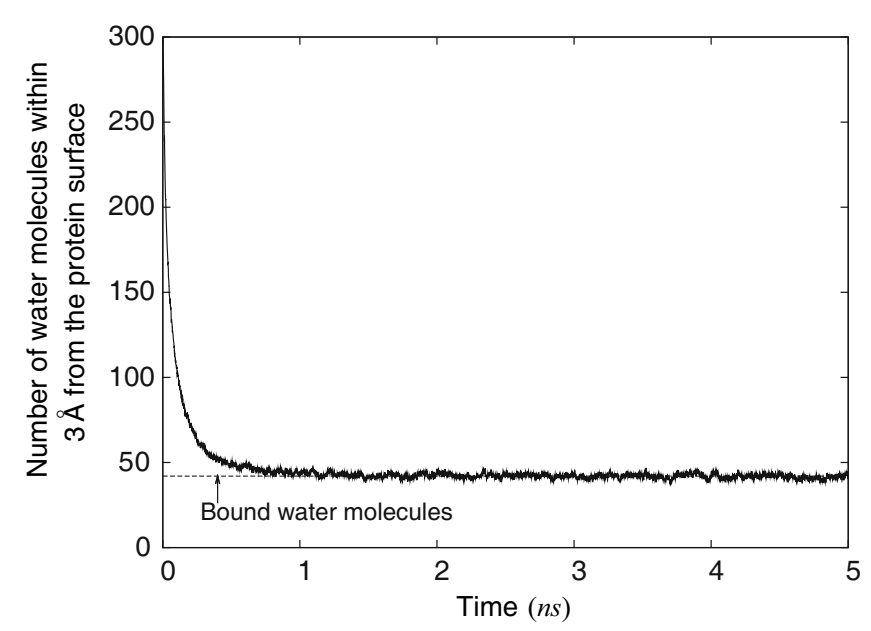

Fig. 6. An example to illustrate the number of water molecules within $3 \AA$ from the protein surface as a function of time.

Table 5. Number of bound water molecules, number of mobile water molecules and their residence times at the protein surface. The bound water molecules have residence times of the order of tens of ns for which good statistics cannot be obtained in a 20 ns simulation.

\begin{tabular}{cccc}
\hline \hline \% trehalose & $N_{\text {bound }}$ & $N_{\text {mobile }}$ & $\tau_{\text {mobile }}(\mathrm{ps})$ \\
\hline 0 & 44 & 207 & 87.64 \\
10 & 26 & 168 & 145.71 \\
20 & 39 & 145 & 204.64 \\
30 & 42 & 162 & 227.01 \\
\hline \hline
\end{tabular}

instead of eq. (5). However, the $\tau_{\text {bound }}$ was found in the time range of $25-100 \mathrm{~ns}$ which is much longer than the length of the simulation in our study, $20 \mathrm{~ns}$. Thus, our treatment of the slow diffusing water molecules as bound water molecules is appropriate. This distinction between bound and mobile water molecules has been successfully used in literature before to explain the two vastly different dielectric relaxation times observed for water associated with biomolecules $[84,85]$.

Table 5 shows the fitting parameters for eq. (5) for all the simulations. It is particularly encouraging to see that the fast relaxation times are of the order of picoseconds. This result is in good quantitative agreement with values reported in ref. [84]. The slow relaxation times found in this study (not reported here) are of the order of nanoseconds which also agree with those reported in the literature [84].

Forty-four water molecules are bound to the protein in pure water. However, this number drops to 26 for the $10 \%$ trehalose- $90 \%$ water mixture and increases to 39 and 42 for the $20 \%$ and $30 \%$ trehalose in water mixtures, respectively. Clearly, some bound water molecules are replaced by trehalose upon the addition of trehalose to pure water.

Since trehalose is a large molecule compared to water, a protein-trehalose hydrogen bond at a particular site on the surface affects many other surrounding sites. This

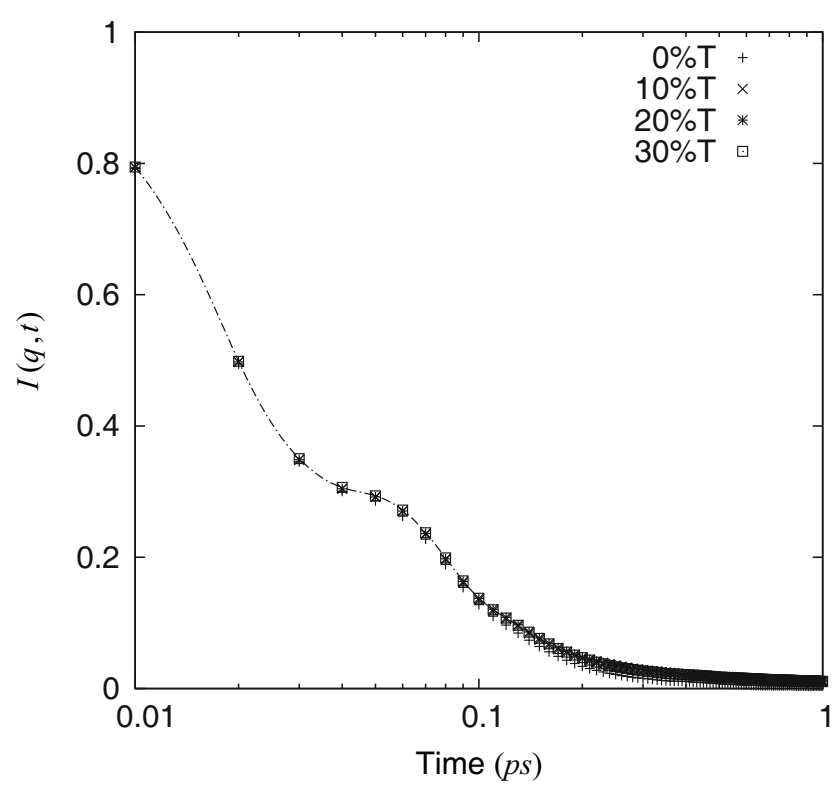

Fig. 7. $I(q, t)$ of water hydrogens that are within $3 \AA$ from the protein surface. A $|\mathbf{q}|$ of $6.28 \AA^{-1}$ was used.

could explain the nearly $40 \%$ drop in the number of bound water molecules in $10 \%$ trehalose.

However, further addition of trehalose leads to an increase in the number of bound water molecules which is roughly compensated by a decrease in the number of mobile water molecules. This trend continues for $30 \%$ trehalose mixture.

In addition, while the number of mobile water molecules in binary mixtures is less than the one in pure water, their relaxation time increases steadily with an increase in trehalose concentration. Clearly, an increase in the concentration of trehalose builds a barrier around the protein that keeps the mobile water molecules closer to the surface of the protein for longer times. This picture is in agreement with the mechanical entrapment model proposed in literature [58].

It is reasonable to expect a similar increase in the relaxation time due to a decrease in temperature, the reason being that at lower temperatures [19] the water molecules are less mobile and, consequently, diffuse more slowly.

In a second study of the hydration layer, we computed the Incoherent Intermediate Scattering function for the water hydrogens at two different length scales: $1 \AA$ and $3.1 \AA$. The first length scale provides information about the relaxation of the internal structure of the layer while the second length scale provides information about the structural relaxation of the layer as a whole. The results are shown in figs. 7 and 8. Figure 7 shows that the relaxation of the internal structure of the layer is insensitive to the presence of trehalose. This is probably due to the time scale of the relaxation which is shorter than $1 \mathrm{ps}$. Moreover, it is interesting to observe the presence of two relaxation processes separated by a plateau at time scales close to $0.1 \mathrm{ps}$. These two processes are independent of trehalose concentration. Figure 8 shows the structural relaxation of the layer as a whole. Note that in this case, 


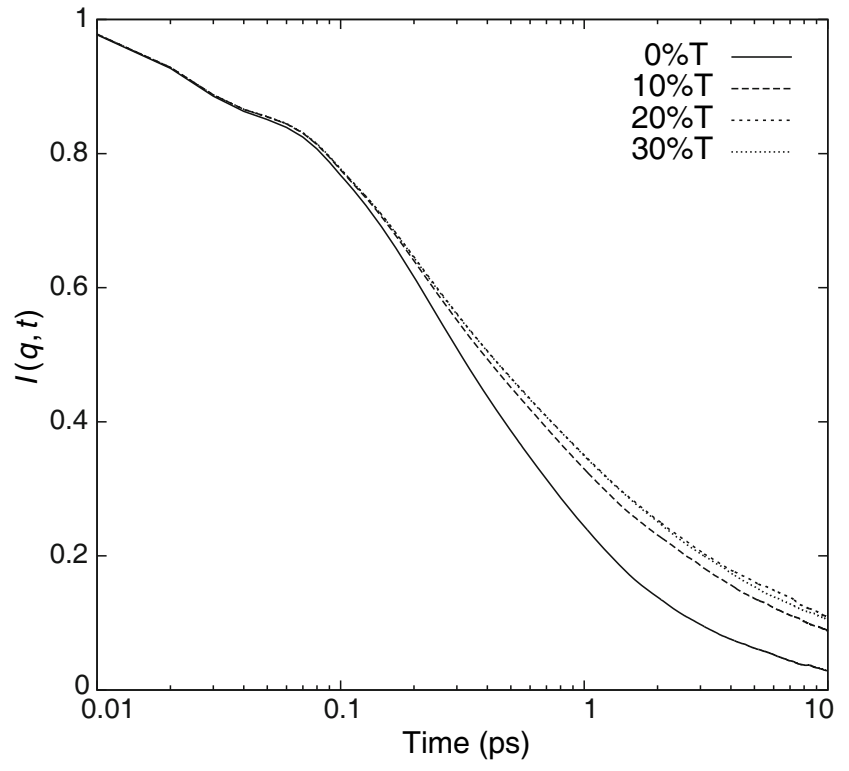

Fig. 8. $I(q, t)$ of water hydrogens that are within $3 \AA$ from the protein surface. A $|\mathbf{q}|$ of $2 \AA^{-1}$ was used.

the relaxation needs one order of magnitude in time more than in the previous case and it is sensitive to the concentration of trehalose. Clearly, trehalose molecules must be displaced for the structure of the whole layer to relax; thus, the higher the concentration of trehalose, the longer the relaxation time.

\subsection{Solvent structure}

The standard protocol to characterize solvent structure is to compute radial distribution functions. However, the evaluation of this type of function becomes very complex in the case of proteins due to the non-spherical nature of the protein surface. Indeed, the computation of the radial distribution function for trehalose or water molecules as a function of the distance from the surface of the protein becomes a computationally challenging task. The reason for this is the calculation of the volume of a shell of thickness $\delta r$ at a distance $r$ from the protein surface, the latter being very irregular. Moreover, this has to be done for every time step in the simulation. Figure 9 shows a simplified representation of the complexity involved in the evaluation of the radial distribution function. Thus, we opted for computing the distribution of solvent molecules around the protein using the formula

$$
G_{i}(r)=\sum_{t=1}^{T} \sum_{i=1}^{N} \frac{n_{i, t}(r)}{T N_{i} 4 \pi r^{2} \delta r},
$$

where, $n_{i, t}(r)$ is the number of solvent molecules between $r+\delta r$ and $r$ from the protein surface at time $t$ and $N_{i}$ is the total number of solvent molecules of type $i, T$ is the total number of time steps in the simulation.

We computed the distribution of water molecules around the protein using the oxygen atoms. Figure 10

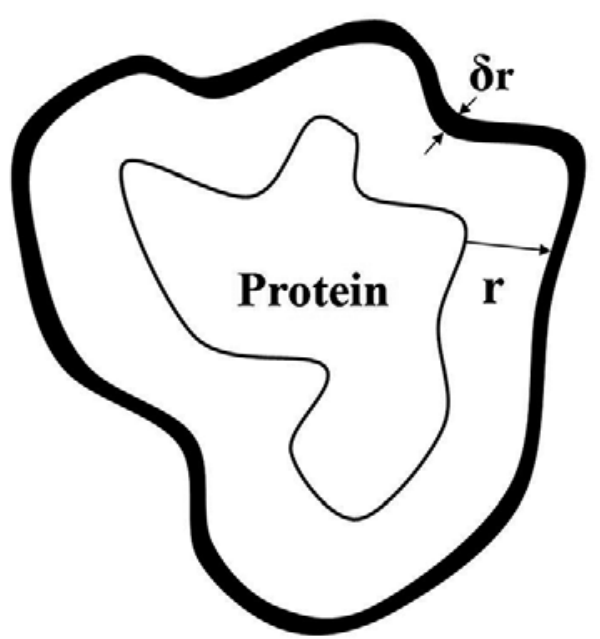

Fig. 9. Cartoon of a shell of water of thickness $\delta r$ at a distance $r$ from the protein surface.

shows the distributions for the three cases analyzed in this paper. Two distinct peaks at $1.91 \AA$ and $2.68 \AA$ from the protein surface are clearly observed. This is in very good quantitative agreement with work by $\mathrm{Gu}$ and Schoenborn [22]. The first peak at $1.91 \AA$ is the best distance for the formation of a hydrogen bond between a water oxygen and a protein hydrogen. On average this is the closest a water oxygen can get to the protein surface. The second peak at $2.68 \AA$ corresponds to a different configuration where a water molecule makes two hydrogen bonds with the protein surface. This places the water hydrogens at a distance $1.91 \AA$ from the protein surface and the oxygen atom attached to them at $2.68 \AA$ from the protein atom nearest to it. Figure 11 illustrates the two stable configurations discussed here.

Figure 10 shows a large increase in the heights of the peaks when the concentration of trehalose increases from $10 \%$ to $20 \%$. However, the increase from $20 \%$ to $30 \%$ trehalose concentration does not change the heights of the peaks significantly. It is possible that the entrapment of a water layer is complete and thus no further increase in the probability of finding water at the surface of the protein is possible.

\subsection{Hydrogen bond analysis}

Proteins interact with the embedding solvents via noncovalent interactions of which hydrogen bonds are known to play a crucial role in determining the dynamics of the protein. We have characterized the formation of solventprotein hydrogen bonds using standard geometric criteria: a hydrogen bond is said to have formed if the distance between the donor and acceptor atoms is less than $3.5 \AA$ and the angle between the donor atom, acceptor hydrogen and acceptor electronegative atom is more than $150^{\circ}$ [19]. The latter is a stricter definition than the $120^{\circ}$ commonly used. 


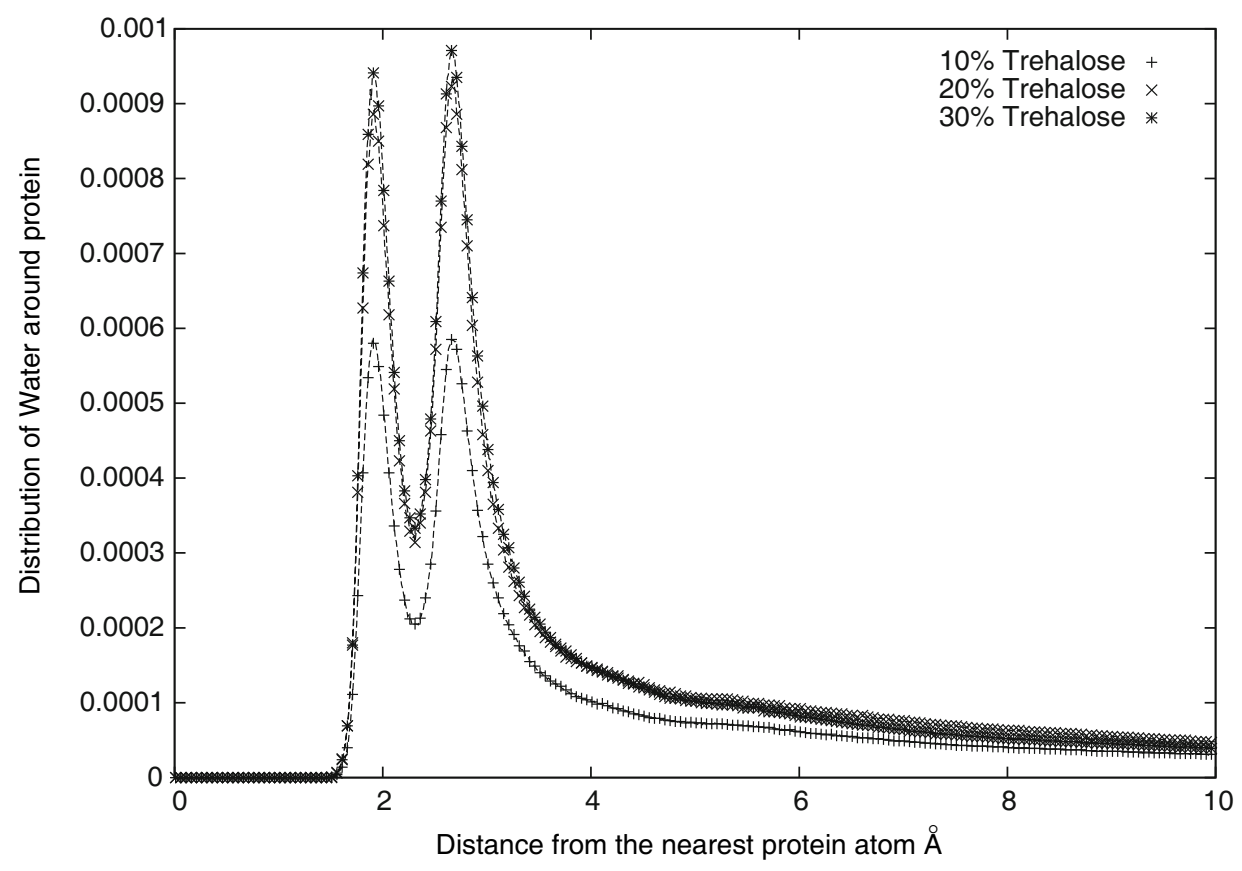

Fig. 10. Distribution of water around the protein surface based on eq. (7).

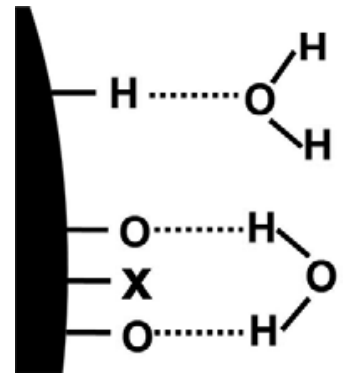

(A)

(B)

Fig. 11. Positions of water molecules that gives rise to the two peaks observed in fig. 10. Configuration A corresponds to the peak at $\sim 1.92 \AA$ while configuration B corresponds to peak at position $\sim 2.68 \AA$ which is the smallest distance between the water oxygen and a protein atom, $X$ in this case.

Table 6 shows the total number of protein-water hydrogen bonds. Note that the numbers of protein-water hydrogen bonds in pure water reported in this study are significantly lower than those reported in ref. [56]. This is due to the different definitions used to determine the formation of hydrogen bonds. Indeed, ref. [56] uses a cutoff for the donor and acceptor distance of $3.4 \AA$ and an angle larger than $120^{\circ}$ as compared to $3.5 \AA$ and $150^{\circ}$ used in this study. Similar values were obtained when we used the cutoffs used in ref. [56].

We know that the SASA of the protein does not change appreciably in binary mixtures. Thus, the area available for the solvent molecules to interact with the protein remains the same. In pure water there are about 196 proteinwater hydrogen bonds. At $10 \%$ trehalose, about 26 of those hydrogen bonds are transferred from water to trehalose. At $20 \%$ trehalose, 37 are transferred to trehalose. As ad-
Table 6. Number of protein-water $(\mathrm{P}-\mathrm{W})$ and protein-trehalose (P-T) hydrogen bonds. Numbers in parenthesis are the errors as measured by the standard deviation.

\begin{tabular}{lll}
\hline \hline$\%$ trehalose & $N_{h}(\mathrm{P}-\mathrm{W})$ & $N_{h}(\mathrm{P}-\mathrm{T})$ \\
\hline 0 & $195.82(9.16)$ & \\
10 & $169.30(12.61)$ & $25.69(5.32)$ \\
20 & $157.32(9.86)$ & $36.96(4.05)$ \\
30 & $167.93(9.6)$ & $24.37(3.55)$ \\
100 & & $90.85(5.34)$ \\
\hline \hline
\end{tabular}

ditional trehalose molecules are put in the solution, one would expect additional transfer of hydrogen bonds from water to trehalose. But the opposite is observed. Only 24 hydrogen bonds are transferred.

It is interesting to note that the total number of hydrogen bonds the protein makes with solvent in all compositions remains around 196 except in pure trehalose where it falls to 91 . This is due to the two reasons: first, the surface area of the protein in pure trehalose is smaller than in the cases of pure water and trehalose-water mixtures and, second, trehalose covers a larger area of the protein surface per hydrogen bond than water.

Using the definition of hydrogen bond previously described, we calculated the hydrogen bond correlation function, $c(t)$, following the methods employed in the literature [23]

$$
c(t)=\frac{\langle h(0) h(t) H(t)\rangle}{\langle h(0) H(0)\rangle},
$$

where $h(t)$ is the hydrogen bond operator which is 1 when a hydrogen bond is present and 0 when it is absent and 


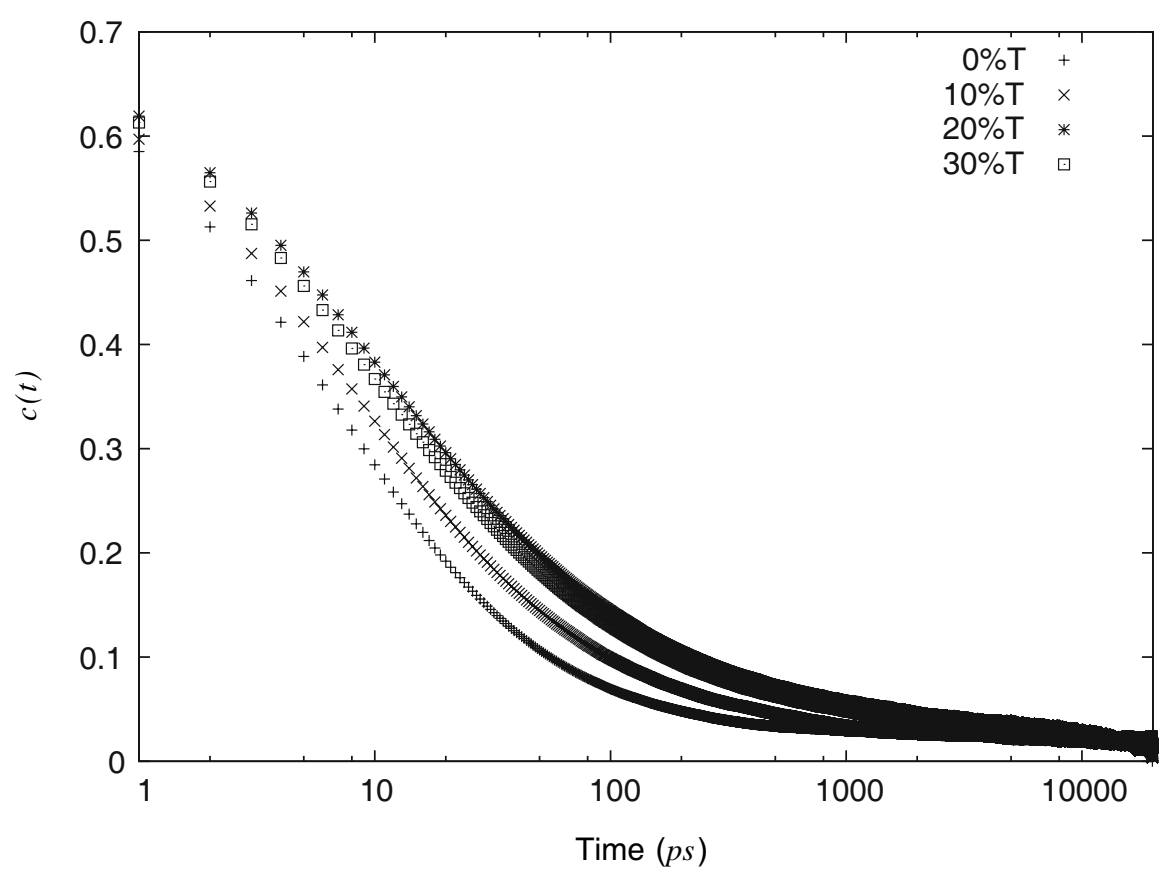

Fig. 12. Hydrogen bond correlation function of protein-water hydrogen bonds in trehalose-water binary solutions.

$H(t)$ is an operator equal to 1 when the atoms involved in the hydrogen bond are within a distance $r$. Essentially, $c(t)$ is the probability that a hydrogen bond formed at time zero exists at time $t$ irrespective of whether it was present or broken in between. Due to the libration and translation of the solvent molecules, this probability decreases to zero quickly. The hydrogen bond network relaxation time, $\tau_{R}$, is defined as the time at which $c(t)$ decays to $1 / e[19,21]$ and is different from the average lifetime of a hydrogen bond which is defined as the time of uninterrupted existence of a hydrogen bond. A hydrogen bond with a molecule like water is easily broken by rotation and translation of the water molecule. The network relaxation time however, is affected primarily by the translation of water molecules.

The most important pair of molecules to be studied is the protein-water pair. Figure 12 shows that the addition of trehalose to a protein-water system enhances the stability of the average protein-water hydrogen bond. The $\tau_{R}$ of this pair is $5.85 \mathrm{ps}, 7.43 \mathrm{ps}, 11.28 \mathrm{ps}$, and $10 \mathrm{ps}$ for solutions with $0 \%, 10 \%, 20 \%$ and $30 \%$ trehalose concentration in water.

A similar behavior is seen when the temperature of a protein-solvent system is reduced. A decrease in temperature decreases the mobility of molecules in the system and, consequently, the average hydrogen bond has a longer lifetime. This has been shown to be true in ref. [19] for the case of Ribonuclease A in pure water and by $\mathrm{Di}$ rama et al. [57] for the case of Lysozyme in glycerol or in trehalose [18]. Thus, the effect of an increase in trehalose concentration is very similar to that of a decrease in temperature.

The second pair that we studied was the water-water pair. It is known that water molecules form a hydrogen bonded network around the protein [9]. A strong network of water around the protein can potentially shield it from changes in the bulk. In our simulations we have looked at shells of water between 0-3 $\mathrm{A}, 3-6 \AA$ and $6-9 \AA$ from the nearest protein atom. In pure water as well as in trehalosewater binary solutions, the water molecules closest to the protein are the least mobile while the water molecules furthest from the protein surface are the most mobile. For the purpose of illustration, we plot the case of $10 \%$ trehalose90\% water mixture in fig. 13 .

The data obtained for the $0-3 \AA$ shell for the case of the protein in pure water and in trehalose-water binary mixtures show an interesting result. As the trehalose content increases, the water-water hydrogen bonds in this shell relax more slowly pointing to a stronger hydrogen bonded network. This result is shown in fig. 14. This observation is in agreement with the trend seen in the behavior of protein-water hydrogen bonds.

Finally, we focus on the protein-trehalose hydrogen bonds. The value of $\tau_{R}$ for this pair is $1.5 \mathrm{~ns}, 0.6 \mathrm{~ns}$ and $0.47 \mathrm{~ns}$ in $10 \%, 20 \%$ and $30 \%$ by weight trehalose concentration, respectively. Figure 15 shows the results. While the $\tau_{R}$ of protein-trehalose hydrogen bonds decreases with increase in trehalose concentration, the $\tau_{R}$ of proteinwater hydrogen bonds increases.

\section{Conclusions}

As quantified by the root mean square deviation, radius of gyration, inertia tensor, solvent accessible surface area and asphericity, we observe no significant difference in the global structure of protein immersed in pure water, 10, 20 and $30 \%$ wt trehalose. Indeed, the numerical values of $R_{g}$, RMSD, asphericity and SASA are essentially 


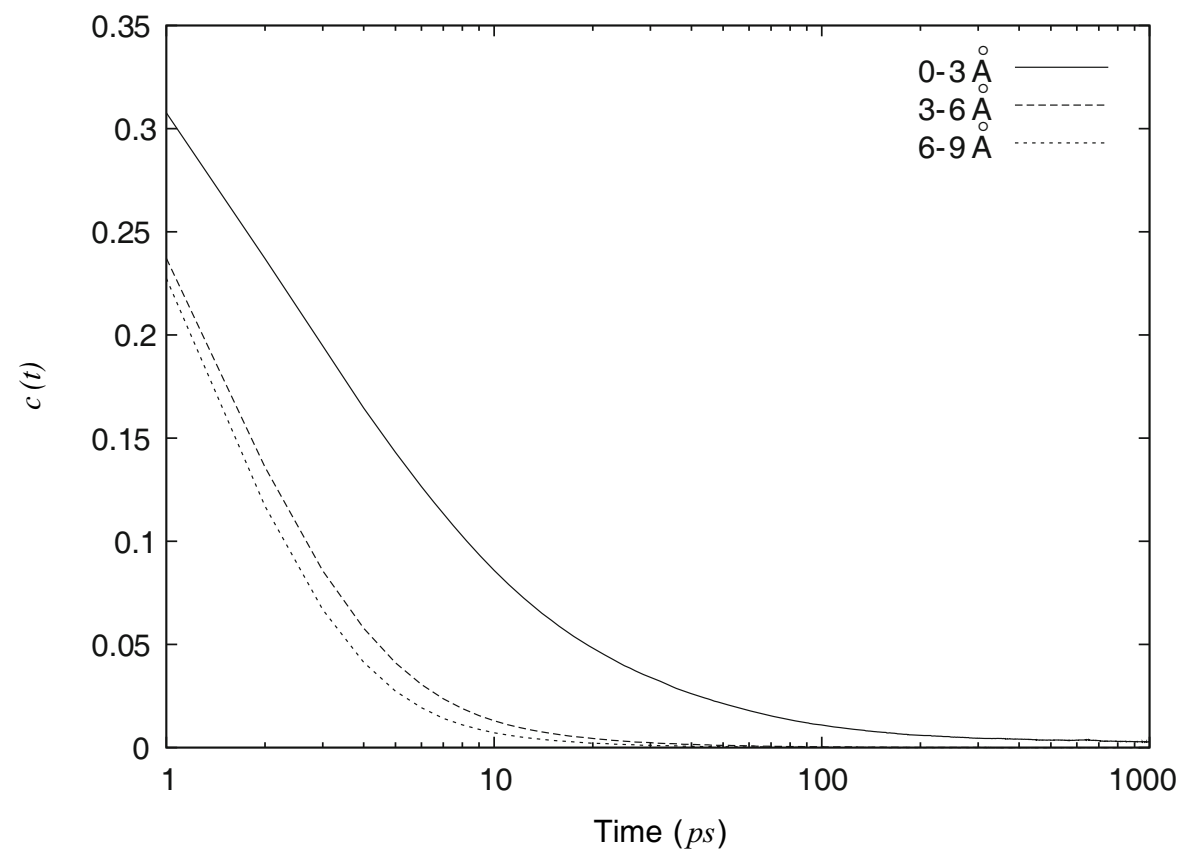

Fig. 13. Hydrogen bond correlation function of various shells of water in $10 \%$ trehalose solution. The shells are defined as the distance from the nearest protein atom.

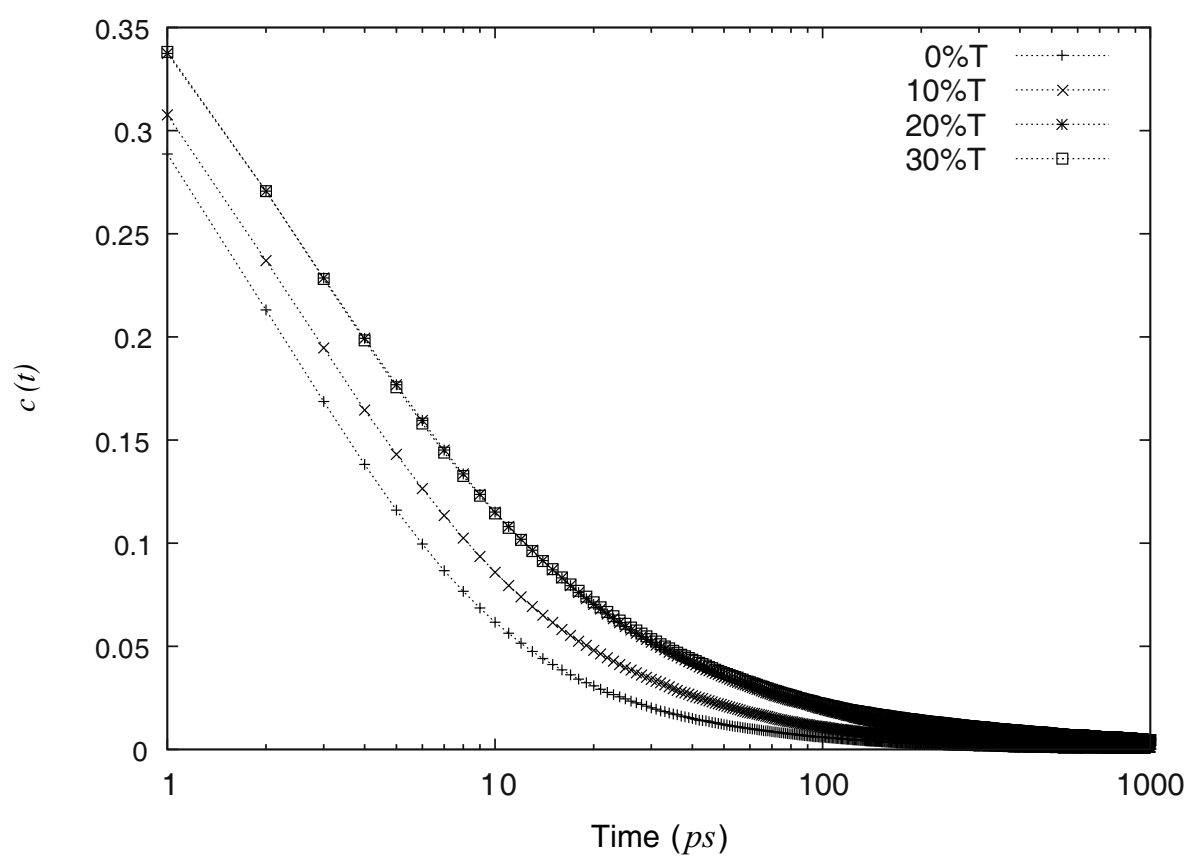

Fig. 14. Hydrogen bond correlation function of the water molecules between $0-3 \AA$ from the protein surface in different solutions.

constant. However, at the local level, the addition of trehalose clearly changes the dielectric constant of the solution making it less polar. This causes the surface residues on the protein to behave differently based on their polarity, spatial position and connectivity to other residues. It is the complex interplay among all of these properties of the residues that leads to their final structures. Thus, the local structure of the protein (e.g. the SASA of each residue) is different from the one in pure water. Notwith- standing the changes at the local level, the fact that the global structure of the protein does not change indicates that the small changes at the residue level somehow cancel each other out. Above 37\% wt trehalose where a percolating hydrogen bond network has been observed in the literature, the behavior can be expected to be quite different. Protein dynamics can be heavily damped and resemble the behavior in pure trehalose. 


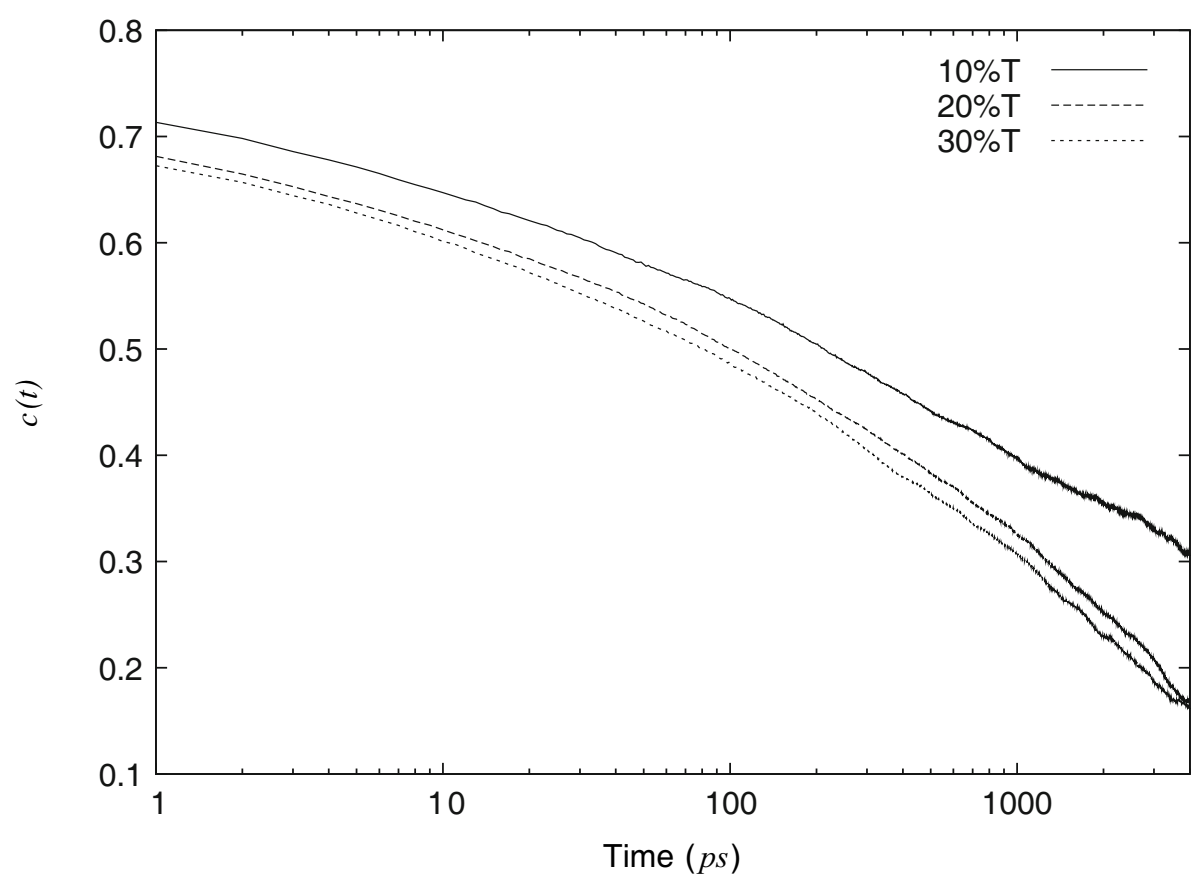

Fig. 15. Hydrogen bond correlation function of protein-trehalose hydrogen bonds in trehalose-water binary solutions.

We studied the global dynamic behavior of the protein using the $I(\mathbf{q}, t)$. Our findings show that the dynamic behavior of the protein slows down with increasing trehalose content. This is true at short time scales, i.e. until about 2 ps. However, for longer time scales we showed that the dynamics of the protein in the $20 \%$ trehalose- $80 \%$ water mixture become slower than in the $30 \%$ trehalose- $70 \%$ water mixture. The origin of this change in the dynamics can be rationalized by studying the number of proteintrehalose hydrogen bonds. In the case of $30 \%$ trehalose$70 \%$ water, there are $35 \%$ fewer protein-trehalose hydrogen bonds than in $20 \%$ trehalose- $80 \%$ water. The translation and libration of the protein involve the displacement of the solvent molecules. When protein atoms are hydrogen bonded to trehalose molecules, their dynamics are slowed down due to the larger inertia of the solvent when compared to a protein-water hydrogen bond. Thus, a higher number of protein-trehalose hydrogen bonds leads to slower dynamics in the protein. The DCCM of the $\alpha$ carbons in the protein sheds light on the local dynamics of the protein which remain largely insensitive to trehalose concentration in binary mixtures.

Pure trehalose, being glassy at $300 \mathrm{~K}$, drastically slows down the protein dynamics. The origin of this slow down is two fold. First, there are more protein-trehalose hydrogen bonds, see table 6 , which slow down the dynamics of the protein due to an increase in inertia. Second, the protein is under isotropic compression as shown by the reduction of its SASA by $8 \%$ when compared to the case of pure water. This decrease, which is caused by burying the highly polar surface residues inside the protein, reduces the space available for the motions of atoms in the protein. Thus, the protein does not have as much freedom to move as in the case of water or water-trehalose binary mixtures.
The equilibrium structure of the hydration shell was studied and the results show the existence of two peaks in the distribution of water around the protein. The peaks occur at distances of $1.91 \AA$ and $2.68 \AA$ from the protein surface and they do not change with changes in the concentration of trehalose. However, their heights do change. Upon an initial addition of trehalose, the heights of the peaks increase until about $20 \%$ trehalose concentration in water. Beyond this concentration the heights remain constant. This was interpreted as the near-complete wetting of protein surface around $20 \%$ trehalose, consequently, no significant change was observed at higher trehalose concentration.

Finally, we also explored the dynamics of the hydration layer at two different length scales: $1 \AA$ and $3.1 \AA$. We found that the dynamics are insensitive to trehalose concentration when explored at short length scales. However, when studied at long length scales, the dynamics of the layer were found to be very sensitive to trehalose concentration.

PKG thanks Dr. Kei Moritsugu and Dr. Edward C. Uberbacher for useful inputs. This work was sponsored by the U.S. DOE under Contract No. DE-AC05-00OR22725 with UT-Battelle, LLC managing contractor for Oak Ridge National Laboratory.

Open Access This is an open access article distributed under the terms of the Creative Commons Attribution License (http://creativecommons.org/licenses/by/3.0), which permits unrestricted use, distribution, and reproduction in any medium, provided the original work is properly cited. 


\section{References}

1. J.H. Crowe, K.A. Madin, T. Am. Microsc. Soc. 93, 513 (1974).

2. J.H. Crowe, J.F. Carpenter, L.M. Crowe, Annu. Rev. Physiol. 60, 73 (1998).

3. J.H. Crowe, L.M. Crowe, A.E. Oliver, N. Tsvetkova, W. Wolkers, F. Tablin, Cryobiology 43, 89 (2001).

4. J.F. Carpenter, J.H. Crowe, Biochem. 28, 3916 (1989).

5. J.F. Carpenter, S.J. Prestrelski, T. Arakawa, Arch. Biochem. Biophys. 303, 456 (1993).

6. J.F. Carpenter, L.M. Crowe, J.H. Crowe, Biochim. Biophys. Acta 923, 109 (1987).

7. R. Mouradian, C. Womersley, L.M. Crowe, J.H. Crowe, Biochim. Biophys. Acta 778, 615 (1984).

8. C. Branca, S. Magazù, G. Maisano, P. Migliardo, J. Chem. Phys. 111, 281 (1999).

9. A. Oleinikova, N. Smolin, I. Brovchenko, A. Geiger, R. Winter, J. Phys. Chem. B 109, 1988 (2005).

10. W. Kauzmann, Adv. Protein Chem. 14, 1 (1959).

11. J.T. Edsall, H.A. McKenzie, Adv. Biophys. 16, 53 (1983).

12. F. Migliardo, S. Magazù, C. Mondelli, J. Mol. Liq. 110, 7 (2004).

13. A. Paciaroni, S. Cinelli, G. Onori, Biophys. J. 83, 1157 (2002).

14. G. Caliskan, D. Mechtani, J.H. Roh, A. Kisliuk, A.P. Sokolov, S. Azzam, M.T. Cicerone, S. Lin-Gibson, I. Peral, J. Chem. Phys. 121, 1978 (2004).

15. H. Frauenfelder, P.W. Fenimore, B.H. Mcmahon, Biophys. Chem. 98, 35 (2002)

16. J.E. Curtis, T.E. Dirama, G.A. Carri, D.J. Tobias, J. Phys. Chem. B 110, 22953 (2006).

17. P.W. Fenimore, H. Frauenfelder, B.H. McMahon, F.G. Parak, Proc. Natl. Acad. Sci. U.S.A. 99, 16047 (2002).

18. T.E. Dirama, J.E. Curtis, G.A. Carri, A.P. Sokolov, J. Chem. Phys. 124, 034901 (2006)

19. M. Tarek, D.J. Tobias, Phys. Rev. Lett. 88, 138101 (2002).

20. A. Paciaroni, Chem. Phys. 292, 397 (2003).

21. F.W. Starr, J.K. Nielsen, H.E. Stanley, Phys. Rev. Lett. 82, 2294 (1999).

22. W. Gu, B.P. Schoenborn, Proteins 22, 20 (1995).

23. A. Luzar, D. Chandler, Phys. Rev. Lett. 76, 928 (1996).

24. A. Luzar, D. Chandler, Nature 379, 55 (1996).

25. P.J. Steinbach, B.R. Brooks, Proc. Natl. Acad. Sci. U.S.A. 90, 9135 (1993)

26. W. Humphrey, A. Dalke, K. Schulten, J. Mol. Graph. 14, 33 (1996).

27. A.R. Bizzarri, S. Cannistraro, Phys. Rev. E 53, R3040 (1996).

28. H. Kovacs, A.E. Mark, W.F. van Gunsteren, Proteins 27, 395 (1997).

29. A.R. Bizzarri, S. Cannistraro, J. Phys. Chem. B 106, 6617 (2002).

30. G.N. Phillips, B.M. Pettitt, Protein Sci. 4, 149 (1995)

31. A. Bonincontro, A. De Francesco, M. Matzeu, G. Onori, A. Santucci, Colloids Surf. B 10, 105 (1997).

32. P. Raiteri, A. Laio, M. Parrinello, Phys. Rev. Lett. 93 087801 (2004).

33. Y. Hayashi, A. Puzenko, Y. Feldman, J. Phys. Chem. B 109, 16979 (2005).

34. S.Y. Noskov, G. Lamoureux, B. Roux, J. Phys. Chem. B 109, 6705 (2005).
35. J.L. Dashnau, N.V. Nucci, K.A. Sharp, J.M. Vanderkooi, J. Phys. Chem. B 110, 13670 (2006).

36. J.L. Dashnau, J.M. Vanderkooi, J. Food Sci. 72, R001 (2007).

37. D.S. Venables, C.A. Schmuttenmaer, J. Chem. Phys. 113 $11222(2000)$.

38. A. Puzenko, Y. Hayashi, Y.E. Ryabov, I. Balin, Y. Feldman, U. Kaatze, R. Behrends, J. Phys. Chem. B 109, 6031 (2005).

39. R. Behrends, K. Fuchs, U. Kaatze, Y. Hayashi, Y. Feldman, J. Chem. Phys. 124, 144512 (2006).

40. A. De Francesco, M. Marconi, S. Cinelli, G. Onori, A. Paciaroni, Biophys. J. 86, 480 (2004).

41. J. Wang, R.M. Wolf, J.W. Caldwell, P.A. Kollman, D.A. Case, J. Comput. Chem. 25, 1157 (2004).

42. V. Réat, R. Dunn, M. Ferrand, J.L. Finney, R.M. Daniel, J.C. Smith, Proc. Natl. Acad. Sci. U.S.A. 97, 9961 (2000).

43. M. Tarek, D. Tobias, Eur. Biophys. J. 37, 701 (2008).

44. K. Gekko, S.N. Timasheff, Biochemistry 20, 4677 (1981).

45. K. Gekko, S.N. Timasheff, Biochemistry 20, 4667 (1981).

46. J.M. Hey, D.R. Macfarlane, Cryobiology 37, 119 (1998).

47. J.M. Hey, D.R. Macfarlane, Cryobiology 33, 205 (1996).

48. M. Ferrario, M. Haughney, I.R. Mcdonald, M.L. Klein, J. Chem. Phys. 93, 5156 (1990).

49. M. Tarek, D.J. Tobias, Biophys. J. 79, 3244 (2000).

50. A. Oleinikova, I. Brovchenko, Mol. Phys. 104, 3841 (2006).

51. W.L. Jorgensen, J. Chandrasekhar, J.D. Madura, R.W. Impey, M.L. Klein, J. Chem. Phys. 79, 926 (1983).

52. R. Abseher, H. Schreiber, O. Steinhauser, Proteins 25, 366 (1996).

53. I.J. Finkelstein, A.M. Massari, M.D. Fayer, Biophys. J. 92, 3652 (2007).

54. C. Atilgan, A.O. Aykut, A.R. Atilgan, Biophys. J. 94, 79 (2008).

55. A.L. Tournier, J. Xu, J.C. Smith, Biophys. J. 85, 1871 (2003).

56. A. Lerbret, P. Bordat, F. Affouard, A. Hédoux, Y. Guinet, M. Descamps, J. Phys. Chem. B 111, 9410 (2007).

57. T.E. Dirama, G.A. Carri, A.P. Sokolov, J. Chem. Phys. 122, 244910 (2005).

58. R.D. Lins, C.S. Pereira, P.H. Hünenberger, Proteins 55, 177 (2004).

59. G. Bellavia, S. Giuffrida, G. Cottone, A. Cupane, L. Cordone, J. Phys. Chem. B 115, 6340 (2011).

60. G. Bellavia, L. Cordone, A. Cupane, J. Therm. Anal. Calorim. 95, 699 (2009).

61. J. Crowe, in Molecular Aspects of the Stress Response: Chaperones, Membranes and Networks, Advances in Experimental Medicine and Biology, Vol. 594, edited by P. Csermely, L. Vígh (Springer, New York, 2007) pp. 143-158.

62. O. Kandror, A. DeLeon, A. Goldberg, Proc. Natl. Acad. Sci. U.S.A. 99, 9727 (2002).

63. A. Lerbret, F. Affouard, A. Hédoux, S. Krenzlin, J. Siepmann, M.-C. Bellissent-Funel, M. Descamps, J. Phys. Chem. B 116, 11103 (2012).

64. M.C. Vaney, S. Maignan, M. Riès-Kautt, A. Ducriux, Acta Crystallogr. D: Biol. Crystallogr. 52, 505 (1996).

65. J. Purvis, L. Yomano, L. Ingram, Appl. Environ. Microbiol. 71, 3761 (2005).

66. D.A. Case, T.A. Darden, T.E. Cheatham III, C.L. Simmerling, J. Wang, R.E. Duke, R. Luo, K.M. Merz, D.A. Pearlman, M. Crowley, R.C. Walker, W. Zhang, B. Wang, S. Hayik, A. Roitberg, G. Seabra, K.F. Wong, F. Paesani, 
X. Wu, S. Brozell, V. Tsui, H. Gohlke, L. Yang, C. Tan, J. Mongan, V. Hornak, G. Cui, P. Beroza, D.H. Mathews, C. Schafmeister, W.S. Ross, P.A. Kollman, AMBER9 (University of California, San Fransisco, 2006).

67. V. Hornak, R. Abel, A. Okur, B. Strockbine, A. Roitberg, C. Simmerling, Proteins 65, 712 (2006).

68. T. Darden, D. York, L. Pedersen, J. Chem. Phys. 98, 10089 (1993).

69. H. Berendsen, J. Postma, W. van Gunsteren, A. DiNola, J. Haak, J. Chem. Phys. 81, 3684 (1984).

70. R. Pastor, B. Brooks, A. Szabo, Mol. Phys. 65, 1409 (1988).

71. W. Mattice, U. Suter, Conformational Theory of Large Molecules (J. Wiley, New York, 1994).

72. H. Goldstein, Classical Mechanics, 2nd edition (AddisonWesley, Reading, 1980).

73. G.A. Arteca, M. Paulino, C.T. Reimann, O. Tapia, Phys. Chem. Chem. Phys. 2, 5259 (2000).

74. D. Frishman, P. Argos, Proteins 23, 566 (1995).

75. A. Anopchenko, T. Psurek, D. Vanderhart, J.F. Douglas, J. Obrzut, Phys. Rev. E 74, 031501 (2006).
76. M. Tarek, G. Martyna, D. Tobias, J. Am. Chem. Soc. 122, $10450(2000)$.

77. J.H. Roh, V.N. Novikov, R.B. Gregory, J.E. Curtis, Z. Chowdhuri, A.P. Sokolov, Phys. Rev. Lett. 95, 038101 (2005).

78. J.H. Roh, J.E. Curtis, S. Azzam, V.N. Novikov, I. Peral, Z. Chowdhuri, R.B. Gregory, A.P. Sokolov, Biophys. J. 91, 2573 (2006).

79. M. Levitt, C. Sander, P.S. Stern, J. Mol. Biol. 181, 423 (1985).

80. W.E. Harte, S. Swaminathan, M.M. Mansuri, J.C. Martin, I.E. Rosenberg, D.L. Beveridge, Proc. Natl. Acad. Sci. U.S.A. 87, 8864 (1990).

81. S. Swaminathan, W.E. Harte, D.L. Beveridge, J. Am. Chem. Soc. 113, 2717 (1991).

82. O. Keskin, S.R. Durell, I. Bahar, R.L. Jernigan, D.G. Covell, Biophys. J. 83, 663 (2002).

83. P.K. Agarwal, Proteins 56, 449 (2004).

84. N. Nandi, B. Bagchi, J. Phys. Chem. B 101, 10954 (1997).

85. S.C. Harvey, P. Hoekstra, J. Phys. Chem. 76, 2987 (1972). 